\title{
Synthesis of Bis-2,3-dihydroquinazolin-4(1H)-ones and 2,3-Dihydroquinazolin-4(1H)-ones Derivatives with the Aid of Silica-Supported Preyssler Nanoparticles
}

\author{
Ali Gharib, ${ }^{1,2}$ Bibi Robabeh Hashemipour Khorasani, ${ }^{2}$ Manouchehr Jahangir, ${ }^{1}$ \\ Mina Roshani, ${ }^{1}$ and Reza Safaee ${ }^{2}$ \\ ${ }^{1}$ Department of Chemistry, Islamic Azad University, Mashhad, Iran \\ ${ }^{2}$ Agricultural Researches and Services Center, Mashhad, Iran
}

Correspondence should be addressed to Ali Gharib; organiccatalyst2008@gmail.com

Received 28 April 2013; Revised 8 June 2013; Accepted 9 June 2013

Academic Editor: Ashraf Aly Shehata

Copyright (C) 2013 Ali Gharib et al. This is an open access article distributed under the Creative Commons Attribution License, which permits unrestricted use, distribution, and reproduction in any medium, provided the original work is properly cited.

One-pot three-component condensation of isatoic anhydride with primary amines or ammonium carbonate and aromatic aldehydes in refluxing ethanol in the presence of catalytic amounts of silica-supported preyssler nanoparticles (SPNP) afforded the corresponding 2,3-dihydroquinazolin-4(1H)-ones in high yields, and bis-dihydroquinazolinones were synthesized for the first time by a novel pseudo-five-component condensation of isatoic anhydride, a primary amine, and a dialdehyde in water. The catalyst is reusable and can be applied several times without any decrease in product yield.

\section{Introduction}

One-pot transformations, particularlymulticomponent reactions (MCRs), are of current interest by Salehi et al. [1]. Since the first MCR reported in 1850 by Strecker [2], this methodology has emerged as an especially attractive synthetic strategy for rapid and efficient library generation due to the fact that the products are formed in a single step and diversity can be achieved simply by varying the reaction components by Strecker [2]. MCRs leading to interesting heterocyclic scaffolds are particularly useful for the creation of diverse chemical libraries of drug-like molecules for biological screening by Domling [3]. 2,3-Dihydroquinazolinone derivatives are an important class of fused heterocycles that display a wide range of biological, pharmacological, and medicinal properties involving antitumor, antibiotic, antipyretic, analgesic, antihypertonic, diuretic, antihistamine, antidepressant, and vasodilating activities by Sadanandam et al. [4]. In addition, 2,3-dihydroquinazolinones have been shown to act as potent tubulin inhibitors with impressive antiproliferative activity against several human cancer cell lines by Chinigo et al.
[5]. Furthermore, these compounds can act analogously to the antimitotic agent colchicine [6]. Additionally, these compounds can easily be oxidized to their quinazolin-4(3H)one analogues by Baker et al. [7], which are themselves important biologically active heterocyclic compounds, Moore et al. [8]. The usual procedure for the preparation of 2,3dihydroquinazolin-4 $(1 H)$-ones involves condensation of the appropriate derivatives of anthranilamide with an aldehyde or ketone using $p$-toluenesulfonic acid as a catalyst under vigorous conditions, by Ozaki et al. [9]. Similar reactions have been reported to proceed under basic conditions, by Kornet [10]. This procedure affords dihydroquinazolinones in good yields but requires long reaction times. The three-step synthesis starting from isatoic anhydride or an anthranilic acid has been reported by Shi et al. [11] and other methods such as reductive cyclisation of $o$-nitrobenzamides with aldehydes and ketones by Steiger et al. [12], reaction of isatoic anhydride with schiff bases, by Li et al. [13], and reduction of quinazolin-4(3H)-ones, by Gorla et al. [14], are also reported for the synthesis of these compounds. Over the last decade, due to the unique properties of nanoparticles 


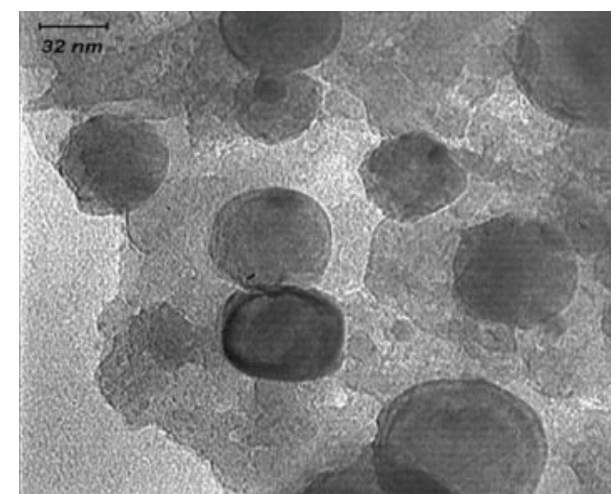

FIGURE 1: TEM image of the synthesized nano- $\mathrm{SiO}_{2}$.

along with their novel properties and potential applications in different fields [14], the synthesis and characterization of catalysts with lower dimension has become an active topic of research. As the particle size decreases, the relative number of surface atoms increases, and thus activity increases. Moreover, due to quantum size effects, nanometersized particles may exhibit unique properties for a wide range of applications, by Martikainen and Stoof [15]. Along this line, polyoxometalates (POMs) are attracting much attention as building blocks for functional composite materials because of their interesting nanosized structures, by Ding et al. [16]. They are ideal models for the construction of hybrid systems, so they are regarded as the potential candidates to be transformed into nanometer-sized materials. In recent years, considerable effort has been devoted to the design and controlled fabrication of nanostructured POMs for using in green reactions. This interest has resulted in the development of numerous protocols for the synthesis of nanostructured materials over a range of sizes. Therefore, the field of nanoPOMs and their applications continue to attract significant attention, so the number of publications and patents continue to grow, and new researchers are entering the field. Thus, plenty of room exists for expanding the exploration of the opportunities for these materials and further exploring, so developing new POMs is still a challenge for POM chemistry. However, in spite of extensive investigations on synthesis and characterization of Keggin-type nanocatalysts, by Sawant et al. [17], the synthesis of sodium 30-tungstopentaphosphate nanocatalysts has been largely overlooked. A Preyssler acid is a highly acidic catalyst with excellent catalytic activity in a variety of acid catalyzed reactions, elsewhere $[18,19]$. The catalyst consists of an anion with a formula of $\left[\mathrm{NaP}_{5} \mathrm{~W}_{30} \mathrm{O}_{110}\right]^{14-}$ which has an unusual five-fold symmetry achieved by fusion of five $\mathrm{PW}_{6} \mathrm{O}_{22}$ groups. The central sodium ion lies not on the equator of the anion but in a plane roughly defined by oxygen atoms of the phosphate groups. The presence of the sodium cation reduces the overall anion symmetry from $D_{5 h}$ to $C_{5 \mathrm{v}}$, by Müller et al. [20]. Silica-supported preyssler nanostructures were obtained through a microemulsion method. Although this procedure has been reported previously, this method has never been reported for the synthesis of Preyssler nanostructures with different morphologies.

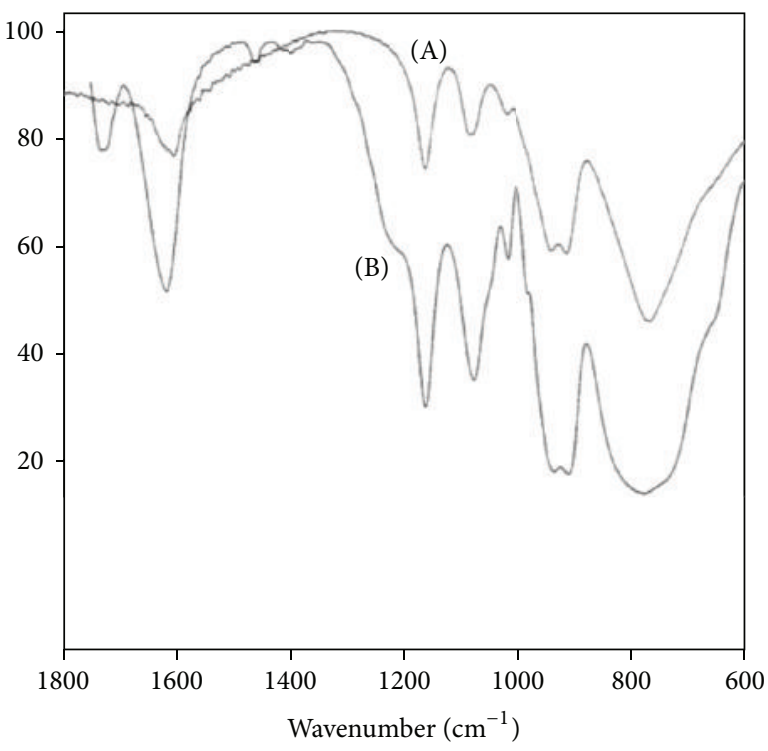

FIGURE 2: Infrared spectroscopy of Preyssler heteropolyacid in bulk form (B) and nanoform (A).

\section{Material and Methods}

All materials were of commercial quality and were used as received. The product purities were determined by GCMS analysis. Mass spectra were recorded on a Shimadzu QP 1100 BX mass spectrometer. Elemental analysis was performed using an electrothermal 9100 apparatus. IR spectra were recorded on $\mathrm{KBr}$ pellets on a Shimadzu IR-470 spectrophotometer. ${ }^{1} \mathrm{H}$ and ${ }^{13} \mathrm{C}$ NMR spectra were determined on a Bruker 300 DRX Avance instrument at $300 \mathrm{MHz}$, respectively.

2.1. Catalyst Preparation. Supported heteropoly acid catalyst was synthesized according to the literature, Alizadeh et al. [21], using a support in powder form $\left(\mathrm{SiO}_{2}\right)$ with an aqueous solution of the heteropolyacids. After stirring the mixture, the solvent was evaporated, dried at $120^{\circ} \mathrm{C}$, and calcined at $250^{\circ} \mathrm{C}$ in a furnace prior to use. Silica-supported Preyssler nanostructures were obtained through the microemulsion method by Heravi et al. [22].

2.2. General Procedure for the Synthesis of 2,3-Dihydroquinazolin-4(1H)-ones. Silica-Supported Preyssler nanoparticles heteropolyacid catalyst $(0.03 \mathrm{mmol})$, isatoic anhydride $(1 \mathrm{mmol})$, primary amine or ammonium acetate $(1.1 \mathrm{mmol})$, and aromatic aldehyde $(1 \mathrm{mmol})$ were added to $5 \mathrm{~mL}$ of water or ethanol and the mixture was stirred in a round bottomed flask at under reflux conditions for the appropriate time (see Tables 1 and 2). After completion of the reaction confirmed by TLC (eluent: $n$-hexane/ethyl acetate: $2 / 1$ ), water was decanted, hot ethanol $(5 \mathrm{~mL})$ was added to the residue which was then filtered. The resulting solution was condensed under reduced pressure. Finally, the crude product was filtered and recrystallized from ethanol. 
TABLE 1: Silica-supported preyssler nanoparticles catalyzed synthesis of 2,3-disubstituted 2,3-dihydroquinazolin-4(1H)-ones derivatives by the reaction of isatoic anhydride with primary amines and aldehydes in water and ethanol under reflux conditions in proper times.

\begin{tabular}{|c|c|c|c|c|c|c|c|c|}
\hline \multirow{2}{*}{ Entry } & \multirow{2}{*}{ Aldehyde (2) } & \multirow{2}{*}{ Amine (3) } & \multirow{2}{*}{ Product (4) } & \multicolumn{2}{|c|}{ Time (h) } & \multicolumn{2}{|c|}{${ }^{\mathrm{a}}$ Yield (\%) } & \multirow{2}{*}{$\mathrm{Mp}\left({ }^{\circ} \mathrm{C}\right)$} \\
\hline & & & & $\mathrm{EtOH}$ & $\mathrm{H}_{2} \mathrm{O}$ & $\mathrm{EtOH}$ & $\mathrm{H}_{2} \mathrm{O}$ & \\
\hline 1 & $\mathrm{HCHO}$ & $\mathrm{NH}_{3}$ & & 2 & 1 & 88 & 92 & $142-145$ \\
\hline 2 & & $\mathrm{C}_{2} \mathrm{H}_{5} \mathrm{NH}_{2}$ & & 4.25 & 2.5 & 83 & 87 & $134-137$ [23] \\
\hline 3 & & $\mathrm{C}_{2} \mathrm{H}_{5} \mathrm{NH}_{2}$ & & 3 & 1 & 85 & 81 & $180-182$ \\
\hline 4 & & & & 5 & 1.5 & 81 & 86 & $214-217[24]$ \\
\hline 5 & & $\mathrm{CH}_{3} \mathrm{NH}_{2}$ & & 2.5 & 1 & 87 & 80.5 & $188-190$ [23] \\
\hline 6 & & $\mathrm{CH}_{3} \mathrm{NH}_{2}$ & & 3 & 1.5 & 72 & 83 & $145-146[23]$ \\
\hline 7 & & $\mathrm{CH}_{3} \mathrm{NH}_{2}$ & & 1.5 & 3.5 & 74 & 87.5 & $164-165$ [23] \\
\hline 8 & & $\begin{array}{l}\mathrm{CH}_{3} \mathrm{CH}_{2} \\
\mathrm{CH}_{2} \mathrm{NH}_{2}\end{array}$ & & 1 & 4.5 & 72 & 82.5 & $135-137$ \\
\hline 9 & & $\begin{array}{l}\mathrm{CH}_{3} \mathrm{CH}_{2} \\
\mathrm{CH}_{2} \mathrm{NH}_{2}\end{array}$ & & 1 & 2.5 & 82.5 & 89.5 & $120-121$ \\
\hline 10 & & & & 4 & 2 & 71 & 78.5 & $214-217$ [34] \\
\hline
\end{tabular}


TABle 1: Continued.

\begin{tabular}{|c|c|c|c|c|c|c|c|c|}
\hline \multirow{2}{*}{ Entry } & \multirow{2}{*}{ Aldehyde (2) } & \multirow{2}{*}{ Amine (3) } & \multirow{2}{*}{ Product (4) } & \multicolumn{2}{|c|}{ Time (h) } & \multicolumn{2}{|c|}{${ }^{\mathrm{a}}$ Yield (\%) } & \multirow{2}{*}{$\mathrm{Mp}\left({ }^{\circ} \mathrm{C}\right)$} \\
\hline & & & & $\mathrm{EtOH}$ & $\mathrm{H}_{2} \mathrm{O}$ & $\mathrm{EtOH}$ & $\mathrm{H}_{2} \mathrm{O}$ & \\
\hline 11 & & & & 2 & 4 & 89 & 95 & $190-192$ \\
\hline 12 & & & & 1.5 & 2.5 & 93 & 97 & $171-172$ \\
\hline 13 & & & & 2 & 4 & 82.5 & 91 & $210-212$ \\
\hline 14 & & & & 1 & 4 & 70 & 81.5 & $195-197$ [24] \\
\hline 15 & & & & 1.5 & 4 & 69 & 80 & $186-188[24]$ \\
\hline 16 & & & & 1 & 3.5 & 68 & 82.5 & $205-208$ [24] \\
\hline 17 & & & & 2.5 & 5 & 82 & 95.5 & $227-228$ \\
\hline 18 & & $\mathrm{C}_{2} \mathrm{H}_{5} \mathrm{NH}_{2}$ & & 1 & 4.5 & 89 & 71.5 & $125-128$ [1] \\
\hline 19 & & $\mathrm{C}_{2} \mathrm{H}_{5} \mathrm{NH}_{2}$ & & 3 & 5 & 82 & 95 & $175-178$ [1] \\
\hline 20 & & $\mathrm{C}_{2} \mathrm{H}_{5} \mathrm{NH}_{2}$ & & 3 & 4.5 & 82 & 96 & $157-160[1]$ \\
\hline
\end{tabular}


TABle 1: Continued.

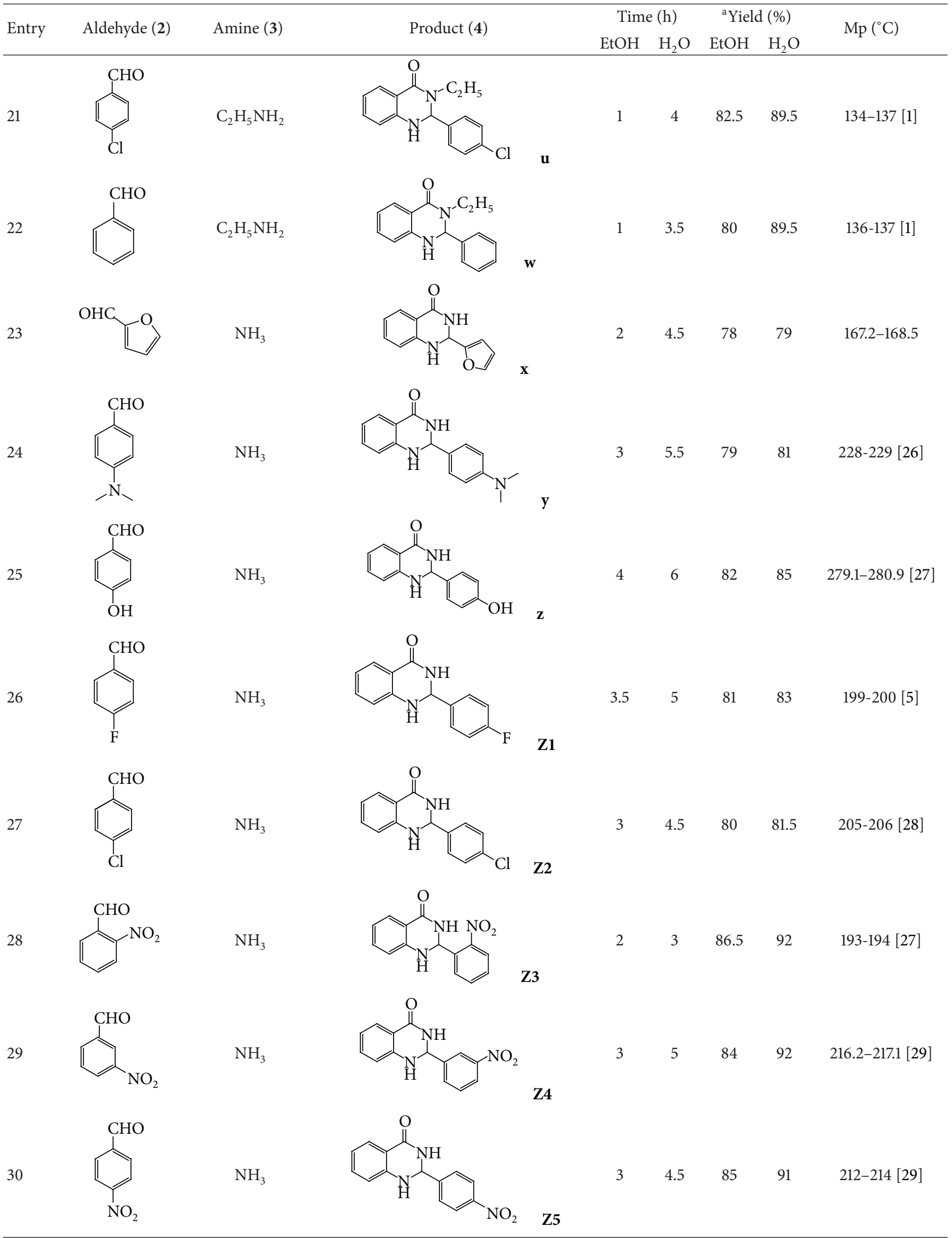


TABle 1: Continued.

\begin{tabular}{|c|c|c|c|c|c|c|c|c|}
\hline \multirow{2}{*}{ Entry } & \multirow{2}{*}{ Aldehyde (2) } & \multirow{2}{*}{ Amine (3) } & \multirow{2}{*}{ Product (4) } & \multicolumn{2}{|c|}{ Time (h) } & \multicolumn{2}{|c|}{${ }^{\mathrm{a}}$ Yield (\%) } & \multirow{2}{*}{$\operatorname{Mp}\left({ }^{\circ} \mathrm{C}\right)$} \\
\hline & & & & $\mathrm{EtOH}$ & $\mathrm{H}_{2} \mathrm{O}$ & $\mathrm{EtOH}$ & $\mathrm{H}_{2} \mathrm{O}$ & \\
\hline 31 & & $\mathrm{NH}_{3}$ & & 5.5 & 4.5 & 76 & 79 & $166-167[30]$ \\
\hline 32 & & $\mathrm{NH}_{3}$ & & 5 & 4 & 78 & 80 & $187-188[31]$ \\
\hline 33 & & $\mathrm{NH}_{3}$ & & 5.5 & 3 & 81 & 83.5 & $249-250[32]$ \\
\hline 34 & & $\mathrm{NH}_{3}$ & & 6 & 3.5 & 80.5 & 82 & $249-250$ \\
\hline 35 & & $\mathrm{NH}_{3}$ & & 4.5 & 3 & 82.5 & 85 & 251, dec. \\
\hline 36 & & $\mathrm{NH}_{3}$ & & 4 & 2.5 & 86 & 92 & $220-221$ \\
\hline 37 & & $\mathrm{NH}_{3}$ & & 6 & 3.5 & 81.5 & 83 & $220-221$ \\
\hline 38 & & $\mathrm{NH}_{3}$ & & 5 & 2.5 & 85 & 89 & $233-234[28]$ \\
\hline 39 & & $\mathrm{NH}_{3}$ & & 5.5 & 4 & 82 & 85 & $266-267$ \\
\hline 40 & & $\mathrm{NH}_{3}$ & & 5 & 3 & 81 & 88 & $188-190$ [33]. \\
\hline
\end{tabular}


TABLe 1: Continued.

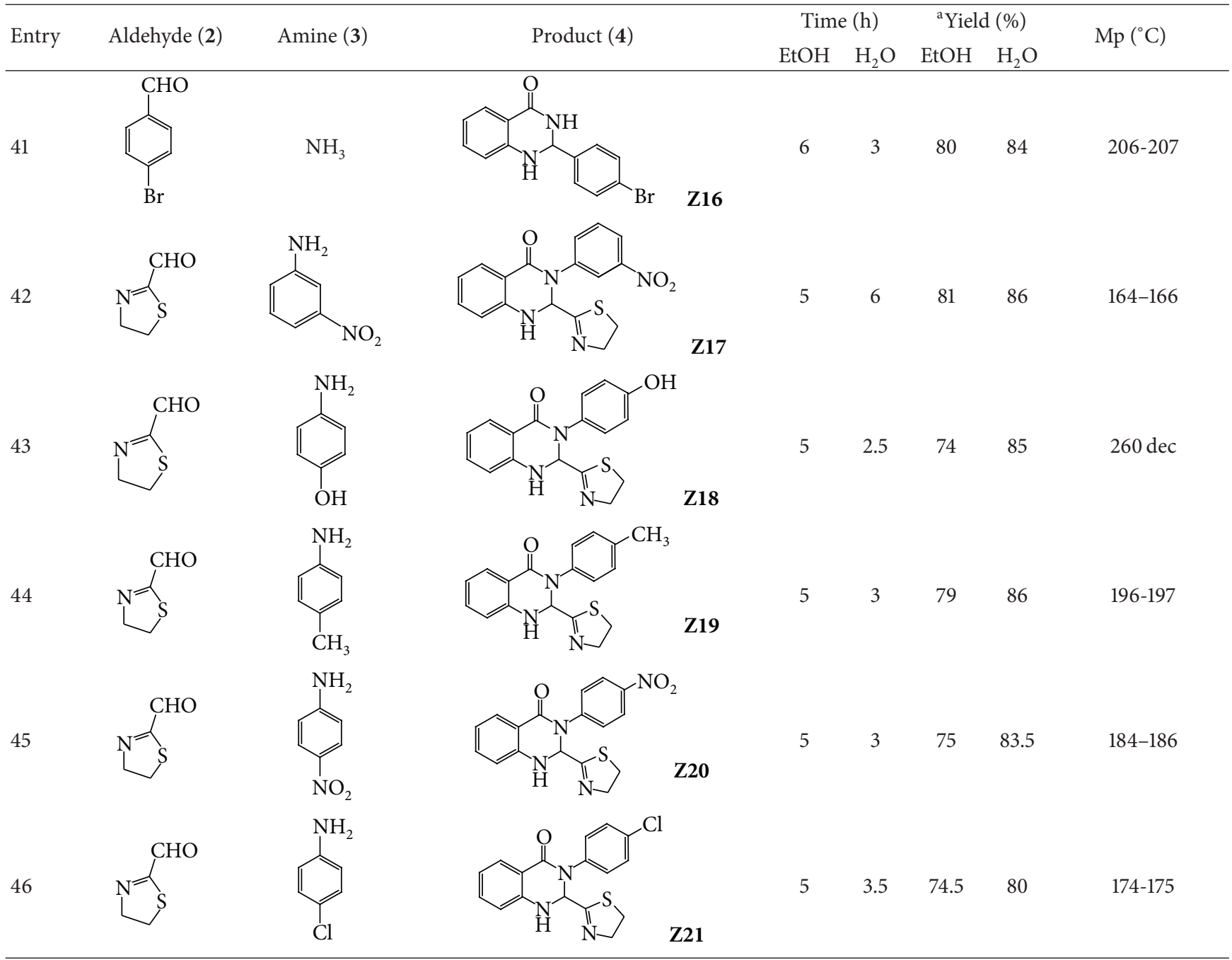

${ }^{\mathrm{a}}$ Isolated yields.

TABLE 2: The conditions of silica nanostructures production via solgel.

\begin{tabular}{lcc}
\hline 4 & $\mathrm{C}_{2} \mathrm{H}_{5} \mathrm{OH} /$ TEOS molar ratio & $\mathrm{A}$ \\
2 & $\mathrm{pH}$ & $\mathrm{B}$ \\
12 & Water/TEOS molar ratio & $\mathrm{C}$ \\
$20 \mathrm{~min}$ & Stirring time & $\mathrm{F}$ \\
$220^{\circ} \mathrm{C}$ & Drying temperature & $\mathrm{G}$ \\
$6 \mathrm{~h}$ & Drying time & $\mathrm{H}$ \\
4 & $\mathrm{C}_{2} \mathrm{H}_{5} \mathrm{OH} / \mathrm{TEOS}$ molar ratio & $\mathrm{A}$ \\
\hline
\end{tabular}

\subsection{Selected Spectroscopic Data}

3-Ethyl-2,3-dihydro-2-(4-hydroxyphenyl)quinazolin-4(1H)-one (4c). IR $\left(\mathrm{KBr}, \mathrm{cm}^{-1}\right): 3440,1680 . \delta_{\mathrm{H}}\left(300 \mathrm{MHz}, \mathrm{CDCl}_{3}\right): 1.00$ $\left(\mathrm{t}, J=7.1 \mathrm{~Hz}, 3 \mathrm{H}, \mathrm{CH}_{3}\right), 2.85(\mathrm{dq}, J=13.6,7.0 \mathrm{~Hz}, 1 \mathrm{H}, \mathrm{CH})$, $3.73(\mathrm{dq}, J=13.6,7.2 \mathrm{~Hz}, 1 \mathrm{H}, \mathrm{CH}), 5.73(\mathrm{~d}, J=1.9 \mathrm{~Hz}, 1 \mathrm{H}$, $\mathrm{CH}), 6.67$ (m, $4 \mathrm{H}, \mathrm{ArH}), 7.14(\mathrm{~m}, 3 \mathrm{H}, \mathrm{ArH}), 7.24$ (d, J = $1.9 \mathrm{~Hz}, 1 \mathrm{H}, \mathrm{NH}), 7.64(\mathrm{dd}, J=7.6,1.4 \mathrm{~Hz}, 1 \mathrm{H}, \mathrm{ArH}), 9.45$ (s, $1 \mathrm{H}, \mathrm{OH}) . \delta_{\mathrm{C}}\left(300 \mathrm{MHz}, \mathrm{CDCl}_{3}\right): 14.5,71.7,115.8,116.4,116.7$,
118.6, 128.9, 129.5, 133.0, 134.4, 148.2, 159.2, 163.3. Anal. Calcd for $\mathrm{C}_{16} \mathrm{H}_{16} \mathrm{~N}_{2} \mathrm{O}_{2}$ : C 72.31, $\mathrm{H}$ 4.62, $\mathrm{N}$ 9.91\%. Found $\mathrm{C} 72.22$, $\mathrm{H}$ 4.71, N 9.82\%. HRMS (EI) Calcd. for $\mathrm{C}_{16} \mathrm{H}_{16} \mathrm{~N}_{2} \mathrm{O}_{2}[\mathrm{M}]^{+}$, 343.1003, Found 343.1008.

2-(4-Nitrophenyl)-3-propyl-2,3-dihydroquinazolin-4(1H)-one (4i). IR $\left(\mathrm{KBr}, \mathrm{cm}^{-1}\right): 3420,1680 . \delta_{\mathrm{H}}\left(300 \mathrm{MHz}, \mathrm{CDCl}_{3}\right)$ $0.94\left(\mathrm{t}, J=7.3 \mathrm{~Hz}, 3 \mathrm{H}, \mathrm{CH}_{3}\right), 1.65\left(\mathrm{~m}, 2 \mathrm{H}, \mathrm{CH}_{2}\right), 2.79$ (ddd, $J=14.1,8.6,5.8 \mathrm{~Hz}, 1 \mathrm{H}, \mathrm{CH}$ ), 4.09 (ddd, $J=13.9,8.7$, $6.6 \mathrm{~Hz}, 1 \mathrm{H}, \mathrm{CH}), 5.86(\mathrm{~s}, 1 \mathrm{H}, \mathrm{CH}), 7.35(\mathrm{~m}, 8 \mathrm{H}, \mathrm{ArH}) . \delta_{\mathrm{C}}$ $\left(300 \mathrm{MHz}, \mathrm{CDCl}_{3}\right)$ 12.7, 22.4, 48.1, 71.6, 116.1, 117.5, 120.9, $125.4,128.6,129.5,134.9,145.5,148.4,149.3,164.2$. Anal. Calcd for $\mathrm{C}_{17} \mathrm{H}_{17} \mathrm{~N}_{3} \mathrm{O}_{3}$ : C, 65.63; H, 5.52; N, 13.56. Found: C, 65.52; $\mathrm{H}, 5.45$; N, 13.49. HRMS (EI) Calcd. for $\mathrm{C}_{17} \mathrm{H}_{17} \mathrm{~N}_{3} \mathrm{O}_{3}[\mathrm{M}]^{+}$, 311.1002, Found 311.1005.

2-(4-Chlorophenyl)-3-(4-isopropylphenyl)-2,3-dihydroquinazolin-4(1H)-one (4k). IR ( $\left.\mathrm{KBr}, \mathrm{cm}^{-1}\right)$ : 3427, 3280, 2964, $1645,1513,1393,1325,1242,990,8825,755 ; \delta_{\mathrm{H}}(300 \mathrm{MHz}$, DMSO-d $) 7.96(1 \mathrm{H}, \mathrm{d}, J=8.4 \mathrm{~Hz}), 7.75(1 \mathrm{H}, \mathrm{d}, J=$ $6.9 \mathrm{~Hz}), 7.64(1 \mathrm{H}, \mathrm{d}, J=2.4 \mathrm{~Hz}), 7.57(1 \mathrm{H}, \mathrm{d}, J=8.4 \mathrm{~Hz})$, 


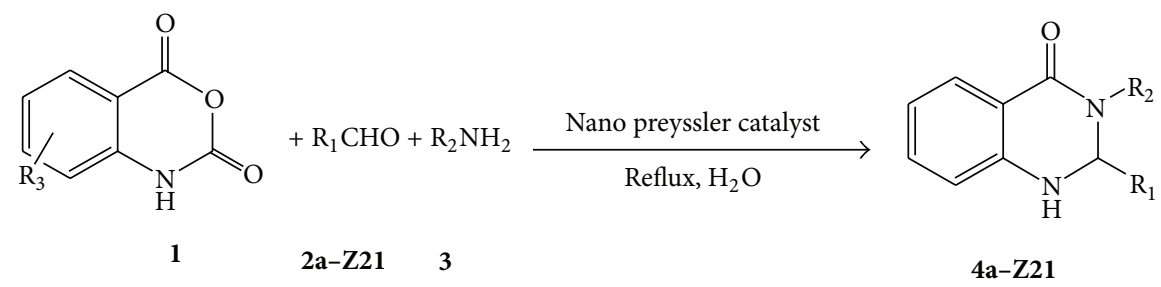

Scheme 1: Synthesized mono- and disubstituted 2,3-dihydroquinazolin-4(1H)-ones using silica-supported preyssler nanoparticlesheteropolyacid under reflux conditions.<smiles>[R]NC(=O)c1ccccc1N</smiles>

Scheme 2: Synthesized disubstituted 2,3-dihydroquinazolin-4(1H)-ones using silica-supported preyssler nanoparticles heteropolyacid under reflux conditions.

$7.26(7 \mathrm{H}, \mathrm{m}), 6.73(2 \mathrm{H}, \mathrm{m}), 6.27(1 \mathrm{H}, \mathrm{d}, J=2.4 \mathrm{~Hz}), 2.87(1 \mathrm{H}$, $\mathrm{m}), 1.18(6 \mathrm{H}, \mathrm{d}, J=6.9 \mathrm{~Hz}) ; \delta_{\mathrm{C}}\left(300 \mathrm{MHz}, \mathrm{DMSO}-d_{6}\right) 162.1$, 146.2, 139.7, 138.5, 133.8, 132.8, 130.3, 128.9, 128.4, 127.2, 126.6, 121.5, 117.7, 115.4, 114.6, 71.7, 32.9, 23.9. HRMS (EI) Calcd. for $\mathrm{C}_{23} \mathrm{H}_{21} \mathrm{ClN}_{2} \mathrm{O}[\mathrm{M}]^{+}$, 376.1000, Found 376.1006; Anal. Calcd for $\mathrm{C}_{23} \mathrm{H}_{21} \mathrm{ClN}_{2} \mathrm{O}$ C 73.30, H 5.62, N 7.43\%. Found C 73.11, H 5.42, N 7.31\%.

3-(4-Isopropylphenyl)-2-(4-methoxyphenyl)-2,3-dihydroquinazolin-4(1H)-one (4l). IR (KBr, cm $\left.{ }^{-1}\right)$ : 3425, 3297, 2955, 1630, $1507,1393,1334,1247,1175,1026,835,705 ; \delta_{\mathrm{H}}(300 \mathrm{MHz}$, DMSO- $\left.d_{6}\right) 7.76(1 \mathrm{H}, \mathrm{d}, J=7.5 \mathrm{~Hz}), 7.55(1 \mathrm{H}, \mathrm{s}), 7.28(7 \mathrm{H}, \mathrm{m})$, $6.85(2 \mathrm{H}, \mathrm{d}, J=8.4 \mathrm{~Hz}), 6.75(2 \mathrm{H}, \mathrm{t}, J=8.4 \mathrm{~Hz}), 6.17(1 \mathrm{H}$, s), $3.74(3 \mathrm{H}, \mathrm{s}), 2.87(1 \mathrm{H}, \mathrm{m}), 1.19(6 \mathrm{H}, \mathrm{d}, J=6.9 \mathrm{~Hz}) ; \delta_{\mathrm{C}}$ $\left(300 \mathrm{MHz}, \mathrm{DMSO}-d_{6}\right) 162.2,159.3,146.5,145.7,138.5,133.6$, $132.9,127.9,127.6,126.4,125.9,117.5,115.4,114.8,113.6,72.4$, 54.9, 32.9, 23.8. HRMS (EI) Calcd. for $\mathrm{C}_{24} \mathrm{H}_{24} \mathrm{~N}_{2} \mathrm{O}_{2}[\mathrm{M}]^{+}$, 372.2003, Found 372.1007. Anal. Calcd for $\mathrm{C}_{24} \mathrm{H}_{24} \mathrm{~N}_{2} \mathrm{O}_{2}$ : C 77.39, H 6.49, N 7.51\%; Found C 77.45, H 6.53, N 7.41\%.

2-(Benzo[d][1,3]dioxol-5-yl)-3-(4-isopropylphenyl)-2,3-dihydroquinazolin-4(1H)-one (4m). IR $\left(\mathrm{KBr}, \mathrm{cm}^{-1}\right): 3438,2965$, $1646,1507,1402,1237,1029,757 ; \delta_{\mathrm{H}}\left(300 \mathrm{MHz}, \mathrm{DMSO}-d_{6}\right)$ $7.75(1 \mathrm{H}, \mathrm{d}, J=7.5 \mathrm{~Hz}), 7.59(1 \mathrm{H}, \mathrm{s}), 7.23(5 \mathrm{H}, \mathrm{m}), 6.96(1 \mathrm{H}, \mathrm{s})$, $6.79(4 \mathrm{H}, \mathrm{m}), 6.19(1 \mathrm{H}, \mathrm{s}), 5.97(2 \mathrm{H}, \mathrm{s}), 2.89(1 \mathrm{H}, \mathrm{m}), 1.17(6 \mathrm{H}$, $\mathrm{d}, J=6.6 \mathrm{~Hz}) ; \delta_{\mathrm{H}}\left(300 \mathrm{MHz}, \mathrm{DMSO}-d_{6}\right) 162.4,147.5,147.2$, $146.4,146.0,138.7,134.7,133.7,127.9,126.5,125.8,119.7,117.5$, $115.6,114.8,107.9,106.7,101.3,72.4,32.9,23.9$. HRMS (EI) Calcd. for $\mathrm{C}_{24} \mathrm{H}_{22} \mathrm{~N}_{2} \mathrm{O}_{3}[\mathrm{M}]^{+}$, 386.2001, Found 386.1006. Anal. Calcd for $\mathrm{C}_{24} \mathrm{H}_{22} \mathrm{~N}_{2} \mathrm{O}_{3}$ : C 74.58, H 5.75, N 7.26\%. Found C 74.42, H 5.81, N 7.37\%.

2,3-Bis(4-methoxyphenyl)-2,3-dihydroquinazolin-4(1H)-one (4q). IR (KBr, cm $\left.{ }^{-1}\right): 3425,2938,2835,1637,1512,1393,1442$, $1245,1176,1027,997,830,764 ; \delta_{\mathrm{H}}\left(300 \mathrm{MHz}, \mathrm{DMSO}-d_{6}\right)$ $7.72(1 \mathrm{H}, \mathrm{d}, J=7.8 \mathrm{~Hz}), 7.42(1 \mathrm{H}, \mathrm{s}), 7.28(3 \mathrm{H}, \mathrm{m}), 7.17(2 \mathrm{H}$, $\mathrm{d}, J=8.7 \mathrm{~Hz}), 6.85(4 \mathrm{H}, \mathrm{m}) 6.77(2 \mathrm{H}, \mathrm{t}, J=7.8 \mathrm{~Hz}), 6.17$ $(1 \mathrm{H}, \mathrm{s}), 3.75(3 \mathrm{H}, \mathrm{s}), 3.70(3 \mathrm{H}, \mathrm{s}) ; \delta_{\mathrm{C}}\left(300 \mathrm{MHz}, \mathrm{DMSO}-d_{6}\right)$
$162.4,159.3,157.5,146.7,133.6,132.9,127.9,127.8,127.4,117.4$, 115.2, 114.6, 113.9, 113.6, 72.9, 55.1, 55.5. HRMS (EI) Calcd. for $\mathrm{C}_{22} \mathrm{H}_{20} \mathrm{~N}_{2} \mathrm{O}_{3}[\mathrm{M}]^{+}, 360.1004$, Found 360.1008. Anal. Calcd for $\mathrm{C}_{22} \mathrm{H}_{20} \mathrm{~N}_{2} \mathrm{O}_{3}$ : C 73.32, H 5.58, N 7.76\%. Found C 73.22, H 5.43, N 7.65\%.

2,3-Dihydro-2-(3-nitrophenyl)-3-(thiazol-2-yl)quinazolin-4(1H)one (4Z17). IR (KBr, cm $\left.\mathrm{cm}^{-1}\right)$ : 3365, 3078, 2962, 1639, 1527, $1505,1445,1392 . \delta_{\mathrm{H}}\left(300 \mathrm{MHz}, \mathrm{DMSO}-d_{6}\right) 7.96(\mathrm{~m}, 10 \mathrm{H}$, Ar-H), 7.50 (d, $J=3.24 \mathrm{~Hz}, 1 \mathrm{H}, \mathrm{CH}), 8.33$ (d, $J=3.24,1 \mathrm{H}$, $\mathrm{NH}) . \delta_{\mathrm{C}}\left(300 \mathrm{MHz}, \mathrm{DMSO}-d_{6}\right) 67.8,114.1,116.2,116.5,119.2$, 121.1, 123.5, 128.5, 130.4, 132.4, 135.7, 137.4, 142.3, 146.8, 148.7, 157.8, 160.9. Anal. Calcd for $\mathrm{C}_{17} \mathrm{H}_{14} \mathrm{~N}_{4} \mathrm{O}_{3} \mathrm{~S}$ : C 57.63, $\mathrm{H} 3.97, \mathrm{~N}$ $15.80 \%$. Found C 57.52, H 3.90, N 15.72\%. HRMS (EI) Calcd. for $\mathrm{C}_{17} \mathrm{H}_{14} \mathrm{~N}_{4} \mathrm{O}_{3} \mathrm{~S}[\mathrm{M}]^{+}$, 360.1002, Found 360.1006.

2,3-Dihydro-2-(4-hydroxyphenyl)-3-(thiazol-2-yl)quinazolin4(1H)-one (4Z18). IR (KBr, cm ${ }^{-1}$ ): 3346, 1638, 1614, 1511, 1453. $\delta_{\mathrm{H}}\left(300 \mathrm{MHz}, \mathrm{DMSO}-d_{6}\right) 7.56(\mathrm{~m}, 10 \mathrm{H}, \mathrm{Ar}-\mathrm{H}), 7.25(\mathrm{~d}, J=$ $3.18 \mathrm{~Hz}, 1 \mathrm{H}, \mathrm{CH}), 8.04(\mathrm{~d}, J=3.2 \mathrm{~Hz}, 1 \mathrm{H}, \mathrm{NH}), 9.45$ (s, $1 \mathrm{H}$, $\mathrm{OH}) . \delta_{\mathrm{C}}\left(400 \mathrm{MHz}, \mathrm{DMSO}-d_{6}\right) 68.3,114.6,115.6,115.4,116.0$, 118.5, 127.4, 128.2, 130.5, 135.7, 137.5, 147.4, 157.5, 158.1, 161.2. Anal. Calcd for $\mathrm{C}_{17} \mathrm{H}_{15} \mathrm{~N}_{3} \mathrm{O}_{2} \mathrm{~S}$ : C 62.75, H 4.65, N 12.92\%. Found $\mathrm{C}$ 62.81, $\mathrm{H}$ 4.73, N 12.82\%. HRMS (EI) Calcd. for $\mathrm{C}_{17} \mathrm{H}_{15} \mathrm{~N}_{3} \mathrm{O}_{2} \mathrm{~S}[\mathrm{M}]^{+}$, 325.1002, Found 325.1005.

2-(4,5-Dihydrothiazol-2-yl)-3-p-tolyl-2,3-dihydroquinazolin4(1H)-one (4Z19). IR ( $\left.\mathrm{KBr}, \mathrm{cm}^{-1}\right)$ : 3406, 3045, 1635, 1507, $1453,1393 . \delta_{\mathrm{H}}\left(300 \mathrm{MHz}, \mathrm{DMSO}-d_{6}\right) 2.15\left(\mathrm{~s}, 3 \mathrm{H}, \mathrm{CH}_{3}\right), 7.65$ (m, 10H, Ar-H), 7.35 (d, $J=3.27,1 \mathrm{H}, \mathrm{CH}), 8.15(\mathrm{~d}, J=3.27 \mathrm{~Hz}$, $1 \mathrm{H}, \mathrm{NH}) . \delta_{\mathrm{C}}\left(300 \mathrm{MHz}, \mathrm{DMSO}-d_{6}\right) 20.8,68.3,114.2,115.8$, $116.4,118.5,126.0,128.6,129.6,135.8,137.1,137.6,137.2,147.4$, 158.6, 161.3. Anal. Calcd for $\mathrm{C}_{18} \mathrm{H}_{17} \mathrm{~N}_{3}$ OS: C 66.85, H 5.31, N $12.98 \%$. Found C 66.80, H 5.22, N 12.80\%. HRMS (EI) Calcd. for $\mathrm{C}_{18} \mathrm{H}_{17} \mathrm{~N}_{3} \mathrm{OS}[\mathrm{M}]^{+}$, 325.1002, Found 325.1005.

2,3-Dihydro-2-(4-nitrophenyl)-3-(thiazol-2-yl)quinazolin-4 (1H)one (4Z20). IR (KBr, $\left.\mathrm{cm}^{-1}\right)$ : 3325, 3103, 1637, 1615, 1510, 1445, 


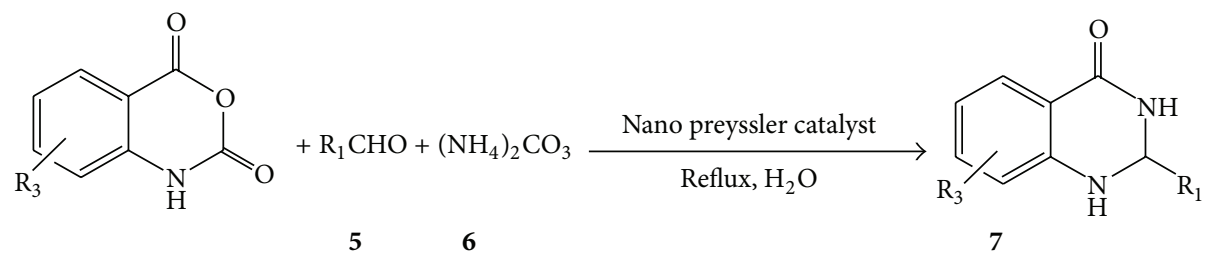

Scheme 3: SynthesisMonosubstituted 2,3-dihydroquinazolin-4(1H)-ones by using ammonium carbonate and silica-supported preyssler nanoparticles heteropolyacid under reflux conditions in water as solvent.

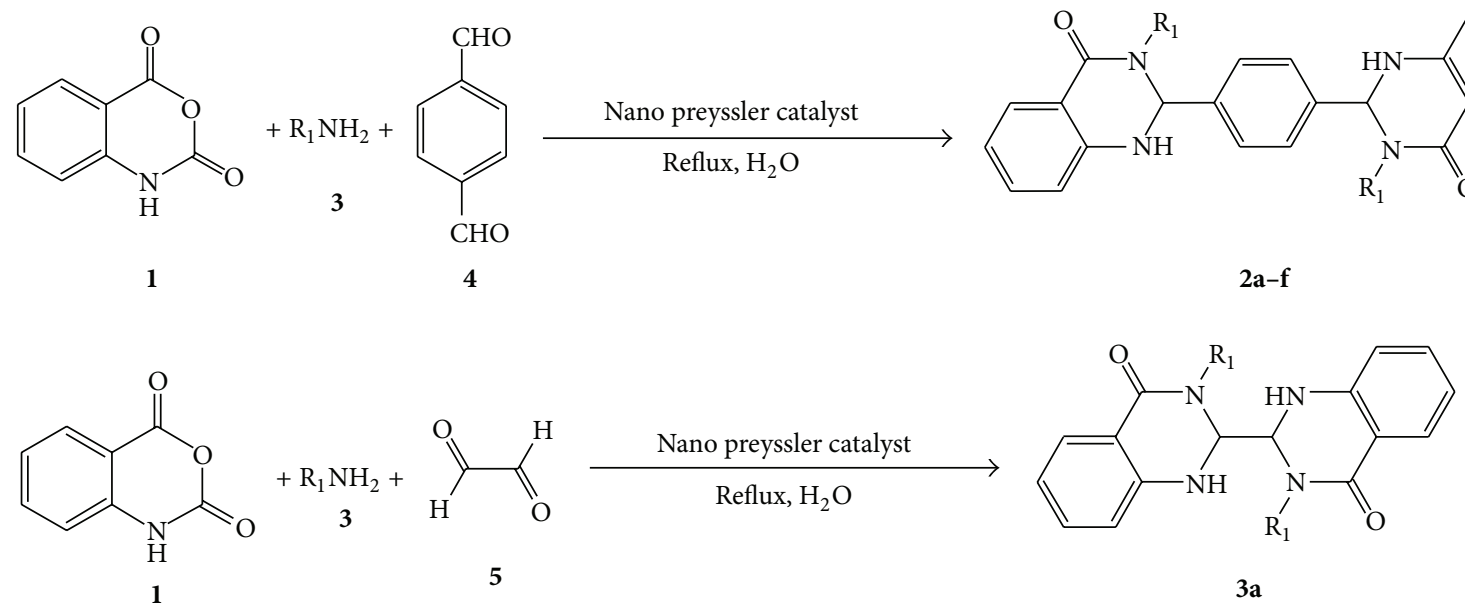

SCHeme 4: Synthesis of Bis-2,3-dihydroquinazolin-4(1H)-ones derivatives using silica-supported preyssler nanoparticles and primary amineand terephthaldehyde under reflux conditions.

1393. $\delta_{\mathrm{H}}\left(300 \mathrm{MHz}, \mathrm{CDCl}_{3}\right) 7.96(\mathrm{~m}, 11 \mathrm{H}, \mathrm{Ar}-\mathrm{H}), 8.24(\mathrm{~s}, 1 \mathrm{H}$, $\mathrm{NH}) . \delta_{\mathrm{C}}\left(300 \mathrm{MHz}, \mathrm{CDCl}_{3}\right) 68.4,114.1,116.1,116.4,119.1,124.2$, $127.4,128.7,135.4,137.7,146.6,147.7,147.7,157.7,160.8$. Anal. Calcd for $\mathrm{C}_{17} \mathrm{H}_{14} \mathrm{~N}_{4} \mathrm{O}_{3} \mathrm{~S}$ : C 66.85, $\mathrm{H}$ 5.31, N 12.98\%. Found C 66.80, H 5.22, N 12.80\%. HRMS (EI) Calcd. for $\mathrm{C}_{17} \mathrm{H}_{14} \mathrm{~N}_{4} \mathrm{O}_{3} \mathrm{~S}$ $[\mathrm{M}]^{+}, 354.1001$, Found 354.1006.

2-(4-Chlorophenyl)-2,3-dihydro-3-(thiazol-2-yl)quinazolin4(1H)-one (4Z21). IR ( $\left.\mathrm{KBr}, \mathrm{cm}^{-1}\right)$ : 3360, 3333, 3078, 1624, $1613,1508,1433 . \delta_{\mathrm{H}}\left(300 \mathrm{MHz}, \mathrm{CDCl}_{3}\right): 7.75(\mathrm{~m}, 11 \mathrm{H}, \mathrm{Ar}-\mathrm{H})$, $8.16(\mathrm{~d}, J=3.72 \mathrm{~Hz}, \mathrm{NH}) . \delta_{\mathrm{C}}\left(300 \mathrm{MHz}, \mathrm{CDCl}_{3}\right): 67.9,114.1$, $116.0,116.1,118.2,128.0,128.6,129.3,133.3,135.5,137.7,139.4$, 146.6, 157.6, 161.1. Anal. Calcd for $\mathrm{C}_{17} \mathrm{H}_{14} \mathrm{ClN}_{3} \mathrm{OS}$ : C, 59.38, $\mathrm{H}$, 4.11, N, 12.22\%. Found C 59.21, H, 4.01, N, 12.11\%. HRMS (EI) Calcd. for $\mathrm{C}_{17} \mathrm{H}_{14} \mathrm{ClN}_{3} \mathrm{OS}[\mathrm{M}]^{+}$, 343.1003, Found 343.1008.

2,3-Dihydro-2-[4(1,2,3,4-tetrahydro-4-oxo-3-p-tolylquinazolin-2-yl)phenyl]-3-p-tolylquinazolin-4(1H)-one (2a). IR ( $\mathrm{KBr}$, $\left.\mathrm{cm}^{-1}\right): 3293,1644 . \delta_{\mathrm{H}}\left(300 \mathrm{MHz}, \mathrm{DMSO}-d_{6}\right) 2.25(\mathrm{~s}, 6 \mathrm{H}$, $\left.\mathrm{CH}_{3}\right), 6.13(\mathrm{~d}, J=1.8 \mathrm{~Hz}, 2 \mathrm{H}, \mathrm{CH}), 7.35-740(\mathrm{~m}, 20 \mathrm{H}, \mathrm{ArH})$, $7.55(\mathrm{~d}, J=1.8 \mathrm{~Hz}, 2 \mathrm{H}, \mathrm{NH}) . \delta_{\mathrm{C}}\left(300 \mathrm{MHz}, \mathrm{DMSO}-d_{6}\right) 20.9$, $72.5,114.7,115.4,117.8,126.4,126.7,127.9,129.2,133.8,135.3$, 138.2, 140.8, 146.5, 162.3. Anal. Calcd for $\mathrm{C}_{36} \mathrm{H}_{30} \mathrm{~N}_{4} \mathrm{O}_{2}$ : C, 78.50; H, 5.50; N, 10.10. Found: C, 78.41; H, 5.45; N, 9.78. HRMS (EI) Calcd. for $\mathrm{C}_{36} \mathrm{H}_{30} \mathrm{~N}_{4} \mathrm{O}_{2}[\mathrm{M}]^{+}, 550.2004$, Found 550.1007 .

3-(4-Chlorophenyl)-2-\{4-[3-(4-chlorophenyl)-1,2,3,4-tetra-hydro-4-oxoquinazolin-2-yl]phenyl\}-2,3-dihydroquinazolin-4(1H)one (2c). IR $\left(\mathrm{KBr}, \mathrm{cm}^{-1}\right): 3322,1616 . \delta_{\mathrm{H}}(300 \mathrm{MHz}, \mathrm{DMSO}-$ $\left.d_{6}\right) 6.18(\mathrm{~s}, 2 \mathrm{H}, \mathrm{CH}), 7.16-7.45(\mathrm{~m}, 22 \mathrm{H}, 20 \times \mathrm{ArH}, 2 \times \mathrm{NH})$. $\delta_{\mathrm{C}}\left(300 \mathrm{MHz}, \mathrm{DMSO}-d_{6}\right) 70.9,113.3,113.4,116.3,125.5,126.5$, $126.7,128.9,132.7,137.9,139.3,145.2,160.8$. Anal. Calcd for $\mathrm{C}_{34} \mathrm{H}_{24} \mathrm{Cl}_{2} \mathrm{~N}_{4} \mathrm{O}_{2}$ : C, 69.0; H, 4.0; N, 9.40. Found C, 68.85. H, 3.89; N, 9.22. HRMS (EI) Calcd. for $\mathrm{C}_{34} \mathrm{H}_{24} \mathrm{Cl}_{2} \mathrm{~N}_{4} \mathrm{O}_{2}[\mathrm{M}]^{+}$, 590.2001 , Found 590.1006.

2,3-Dihydro-2-\{4-[1,2,3,4-tetrahydro-4-oxo-3-(thiazol-2-yl) Quinazolin-2-yl]phenyl\}-3-(thiazol-2-yl)quinazolin-4(1H)one (2d). IR $\left(\mathrm{KBr}, \mathrm{cm}^{-1}\right): 3321,1616 . \delta_{\mathrm{H}}\left(300 \mathrm{MHz}, \mathrm{DMSO}-d_{6}\right)$ 6.94-7.89 (m, 20H). $\delta_{\mathrm{C}}\left(300 \mathrm{MHz}, \mathrm{DMSO}-d_{6}\right)$ 68.2, 114.6, 116.5, 118.8, 125.8, 126.3, 128.6, 128.7, 135.5, 137.9, 139.2, 145.6, 160.8. Anal. Calcd for $\mathrm{C}_{28} \mathrm{H}_{20} \mathrm{~N}_{6} \mathrm{O}_{2} \mathrm{~S}_{2}$ : C, 62.70, H, 3.80; N, 15.64. Found: C, 62.57; H, 3.69; N, 15.58. HRMS (EI) Calcd. for $\mathrm{C}_{28} \mathrm{H}_{20} \mathrm{~N}_{6} \mathrm{O}_{2} \mathrm{~S}_{2}[\mathrm{M}]^{+}$, 536.1004, Found 536.1007.

3-Ethyl-2-[4-(3-ethyl-1,2,3,4-tetrahydro-4-oxoquiazolin-2-yl] phenyl)-2,3-dihydroquinazolin-4(1H)-one (2f). IR ( $\mathrm{KBr}$, $\left.\mathrm{cm}^{-1}\right): 3305,2977,1625 . \delta_{\mathrm{H}}\left(300 \mathrm{MHz}, \mathrm{DMSO}-d_{6}\right) 1.04(\mathrm{t}, J=$ $7.0 \mathrm{~Hz}, 6 \mathrm{H}, \mathrm{CH}_{3}$ ), 2.77 (dt, $\left.J=13.6,7.0 \mathrm{~Hz}, 2 \mathrm{H}, \mathrm{CH}\right), 3.83$ (dt, $J=13.6,7.0 \mathrm{~Hz}, 2 \mathrm{H}, \mathrm{CH}), 5.85(\mathrm{~s}, 2 \mathrm{H}, \mathrm{CH}), 7.05-7.30$ (m, 14 $\mathrm{H}, 12 \times \mathrm{ArH}, 2 \times \mathrm{NH}) . \delta_{\mathrm{C}}\left(300 \mathrm{MHz}, \mathrm{DMSO}-d_{6}\right) 13.9,69.9$, 114.7, 115.5, 117.9, 126.8, 127.9, 133.7, 142.0, 146.6, 162.5. Anal. Calcd for $\mathrm{C}_{22} \mathrm{H}_{26} \mathrm{~N}_{4} \mathrm{O}_{2}$ : C, 73.24; $\mathrm{H}, 6.10 ; \mathrm{N}, 13.12$. Found: $\mathrm{C}$, 73.10; H, 5.98; N, 13.03. HRMS (EI) Calcd. for $\mathrm{C}_{22} \mathrm{H}_{26} \mathrm{~N}_{4} \mathrm{O}_{2}$ $[\mathrm{M}]^{+}, 426.2004$, Found 426.2009.

3-Benzyl-2-[4-(3-benzyl-1,2,3,4-tetrahydro-4-oxoquinazolin2-yl)phenyl]-2,3-dihydroquinazolin-4(1H)-one (2g). IR ( $\mathrm{KBr}$, $\left.\mathrm{cm}^{-1}\right): 3290,1644 . \delta_{\mathrm{H}}\left(300 \mathrm{MHz}, \mathrm{DMSO}-d_{6}\right) 3.77(\mathrm{~d}$, 


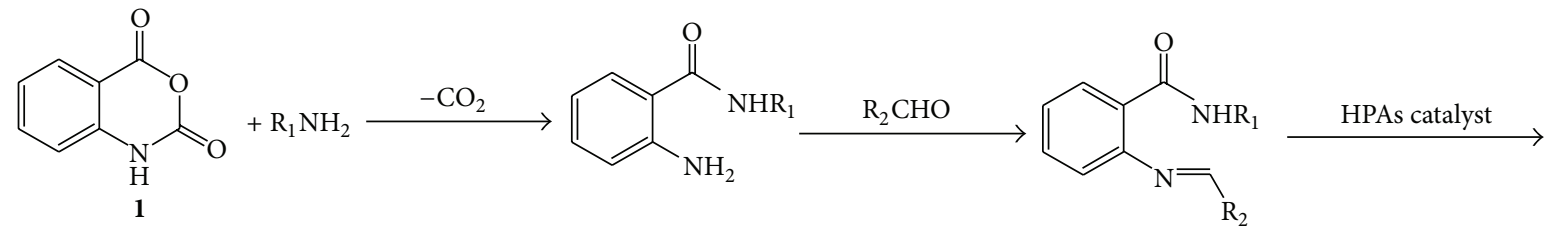

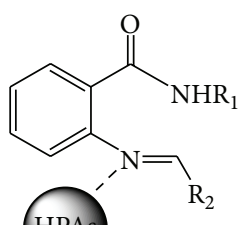<smiles>[R]C1Nc2ccccc2C(=O)N1[R1]</smiles>

$4 a-Z 21$

Scheme 5: The mechanism of synthesized mono- and disubstituted 2,3-dihydroquinazolin-4(1H)-ones using silica-supported preyssler nanoparticles heteropolyacid under reflux conditions.

TABLE 3: Synthesis of 2-Substituted 2,3-dihydroquinazolin-4(1H)-ones derivatives by in the presence of silica-supported preyssler nanoparticles and water as solvent under reflux conditions.

\begin{tabular}{|c|c|c|c|c|}
\hline Entry & Product & Time (h) & ${ }^{\mathrm{a}}$ Yield (\%) & $\mathrm{Mp}\left({ }^{\circ} \mathrm{C}\right)$ \\
\hline 1 & & 4.5 & 80 & $164-165$ [34] \\
\hline 2 & & 4 & 92 & $165-167$ [34] \\
\hline 3 & & 3 & 93 & 233-234 [35] \\
\hline 4 & & 3.5 & 89 & 209-210 [34] \\
\hline 5 & & 3 & 87 & $180-181[36]$ \\
\hline 6 & & 2.5 & 91 & $206-208$ [36] \\
\hline 7 & & 2 & 94.5 & $219-222$ [36] \\
\hline
\end{tabular}

${ }^{\mathrm{a}}$ Isolated yield. 
TABLE 4: Synthesis of bis-2,3-dihydroquinazolin-4(1H)-ones in the presence of Silica-Supported Preyssler Nanoparticles catalyst in water and/or ethanol solvents under reflux conditions.

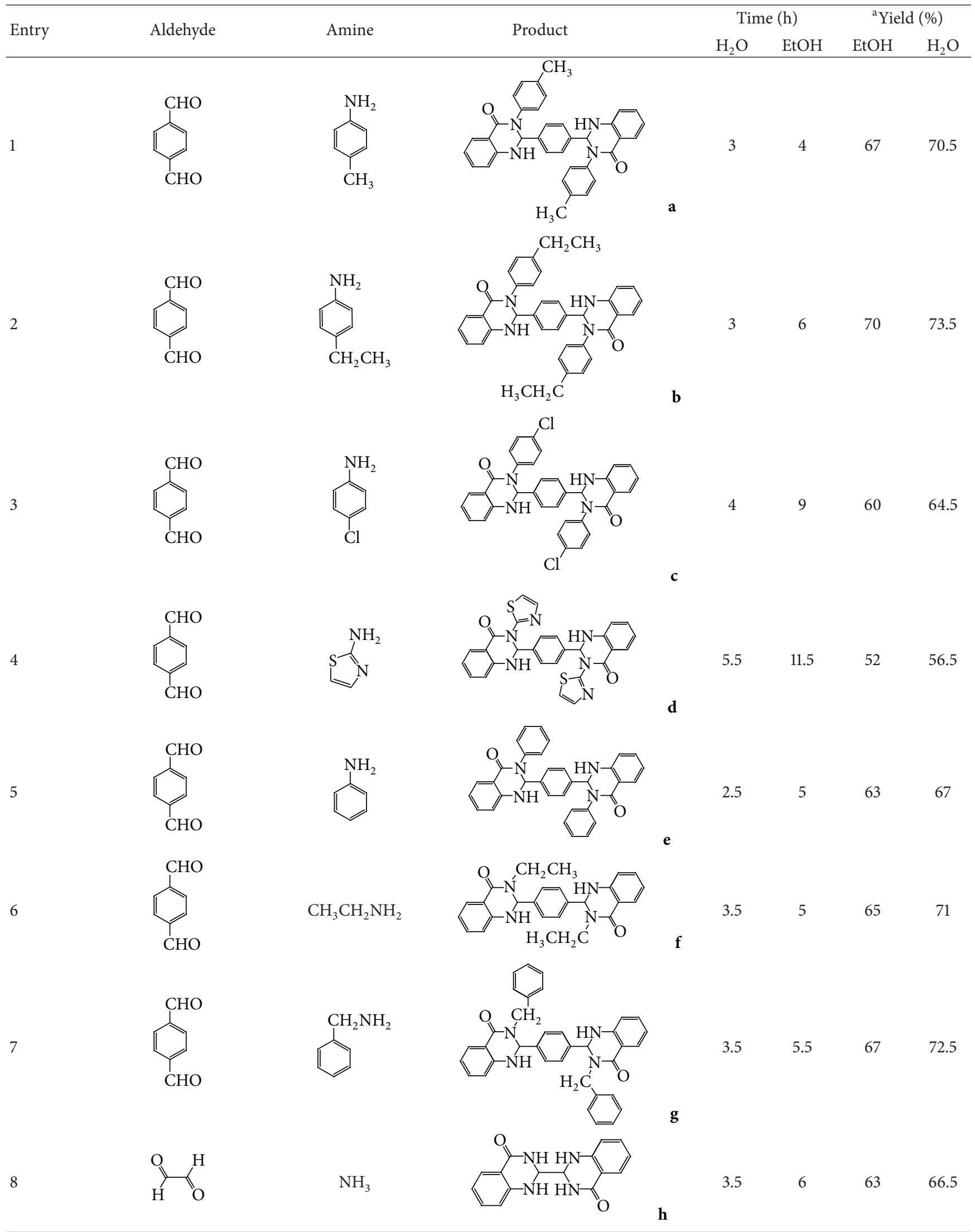


TABLE 4: Continued.

\begin{tabular}{|c|c|c|c|c|c|c|c|}
\hline \multirow{2}{*}{ Entry } & \multirow{2}{*}{ Aldehyde } & \multirow{2}{*}{ Amine } & \multirow{2}{*}{ Product } & \multicolumn{2}{|c|}{ Time (h) } & \multicolumn{2}{|c|}{${ }^{a}$ Yield (\%) } \\
\hline & & & & $\mathrm{H}_{2} \mathrm{O}$ & $\mathrm{EtOH}$ & $\mathrm{EtOH}$ & $\mathrm{H}_{2} \mathrm{O}$ \\
\hline 9 & & $\mathrm{NH}_{3}$ & & 3.5 & 7.5 & 62.5 & 67.5 \\
\hline
\end{tabular}

${ }^{\text {a }}$ Isolated yield.

TABle 5: Solvent effects in the reaction of $\mathrm{C}_{2} \mathrm{H}_{5} \mathrm{NH}_{2}$, isatoic anhydride, and terephthaldehyde for the synthesis of $\left(2,2^{\prime}-(1,4-\right.$ phenylene)bis(3-(4-ethylphenyl)-2,3-dihydroquinazolin-4(1H)-one)) (Table 4, Entry 2, product 2b) in the presence of silica-supported preyssler nanoparticles catalyst.

\begin{tabular}{lccc}
\hline Entry & Solvent & ${ }^{\mathrm{b}}$ Yield (\%) & Time (h) \\
\hline 1 & $\mathrm{H}_{2} \mathrm{O}$ & 73.5 & 3 \\
2 & $\mathrm{TBAB}$ & 61 & 8 \\
3 & $\mathrm{C}_{2} \mathrm{H}_{5} \mathrm{OH}$ & 70 & 6 \\
4 & $\mathrm{CH}_{3} \mathrm{OH}$ & 37 & 12 \\
5 & $\mathrm{CH}_{3} \mathrm{CN}$ & 27 & 20 \\
6 & $\mathrm{CH}_{2} \mathrm{Cl}_{2}$ & - & - \\
7 & $\mathrm{C}_{6} \mathrm{H}_{5} \mathrm{CH}_{3}$ & - & - \\
8 & $\mathrm{CCl}_{4}$ & - & - \\
\hline
\end{tabular}

${ }^{\mathrm{b}}$ Isolated yield.

$J=15.4 \mathrm{~Hz}, 2 \mathrm{H}, \mathrm{CH}), 5.25(\mathrm{~d}, J=15.4 \mathrm{~Hz}, 2 \mathrm{H}, \mathrm{CH}), 5.74(\mathrm{~d}$, $J=2.3 \mathrm{~Hz}, 2 \mathrm{H}, \mathrm{CH}), 7.0-7.56(\mathrm{~m}, 22 \mathrm{H}, \mathrm{ArH}), 7.37(2 \mathrm{H}, \mathrm{d}, J$ $=2.3 \mathrm{~Hz}, \mathrm{NH}) . \delta_{\mathrm{C}}\left(300 \mathrm{MHz}, \mathrm{DMSO}-d_{6}\right) 45.99,68.2,113.5$, $113.4,116.3,125.1,125.9,126.2,126.4,127.6,132.5,136.3,139.8$, 145.0, 161.6. Anal. Calcd for $\mathrm{C}_{36} \mathrm{H}_{30} \mathrm{~N}_{4} \mathrm{O}_{2}$ : C, 78.53; H, 5.56; N, 10.14. Found: C, 78.42; H, 5.45; N, 9.92. HRMS (EI) Calcd. for $\mathrm{C}_{36} \mathrm{H}_{30} \mathrm{~N}_{4} \mathrm{O}_{2}[\mathrm{M}]^{+}, 550.2005$, Found 550.2008.

2,3-Dihydro-2-(1,2,3,4-tetrahydro-4-oxoquinazolin-2-yl)quinazoline-4(1H)-one (3h). IR ( $\left.\mathrm{KBr}, \mathrm{cm}^{-1}\right)$ : 3354, 3300, 1638. $\delta_{\mathrm{H}}\left(300 \mathrm{MHz}, \mathrm{DMSO}-d_{6}\right) 4.83(\mathrm{~m}, 2 \mathrm{H}, \mathrm{CH}), 6.89-7.70(\mathrm{~m}$, $10 \mathrm{H}, 8 \times \mathrm{ArH}, 2 \times \mathrm{NH}), 8.44(2 \mathrm{H}, \mathrm{d}, J=5.3 \mathrm{~Hz}, \mathrm{NH}) . \delta_{\mathrm{C}}$ $\left(300 \mathrm{MHz}, \mathrm{DMSO}-d_{6}\right)$ 67.7, 117.1, 117.5, 118.6, 126.4, 127.3, 132.4, 132.5, 144.9, 167.4. Anal. Calcd for $\mathrm{C}_{16} \mathrm{H}_{14} \mathrm{~N}_{4} \mathrm{O}_{2}$ : C, 65.33; $\mathrm{H}$, 4.82; N, 19.06. Found: C, 65.23; H, 4.71; N, 18.89. HRMS (EI) Calcd. for $\mathrm{C}_{16} \mathrm{H}_{14} \mathrm{~N}_{4} \mathrm{O}_{2}[\mathrm{M}]^{+}$, 294.1004, Found 294.1007.

2,3-Dihydro-2-[4-(1,2,3,4-tetrahydro-4-oxoquinazolin-2-yl)phenyl]quinazolin-4(1H)-one (2i). IR $\left(\mathrm{KBr}, \mathrm{cm}^{-1}\right)$ : 3266, 3185, 1643. $\delta_{\mathrm{H}}\left(300 \mathrm{MHz}, \mathrm{DMSO}-d_{6}\right) 5.75(\mathrm{~d}, J=2.0 \mathrm{~Hz}, 2 \mathrm{H}$, $\mathrm{CH}), 6.67-8.11(\mathrm{~m}, 16 \mathrm{H}, 12 \times \mathrm{ArH}, 4 \times \mathrm{NH}) . \delta_{\mathrm{C}}(300 \mathrm{MHz}$, DMSO- $\left.d_{6}\right): 66.7,114.9,115.7,117.6,127.2,127.8,133.9,142.5$, 148.1, 164.6. Anal. Calcd for $\mathrm{C}_{22} \mathrm{H}_{18} \mathrm{~N}_{4} \mathrm{O}_{2}$ : C, 71.34; $\mathrm{H}, 4.95$; N, 15.16. Found: C, 71.26; H, 4.89; N, 14.98. HRMS (EI) Calcd. for $\mathrm{C}_{22} \mathrm{H}_{18} \mathrm{~N}_{4} \mathrm{O}_{2}[\mathrm{M}]^{+}$, 370.1001, Found 370.1006.

\section{Results and Discussion}

We synthesized mono- and disubstituted 2,3-dihydroquinazolin-4(1H)-ones. Water as solvent resulted in shorter reaction times than ethanol (Table 1). For the synthesis of disubstituted derivatives, isatoic anhydride, a primary amine, and an aromatic aldehyde in the presence of silica-supported preyssler nanoparticles heteropolyacid were reacted in ethanol or water under reflux conditions to afford the expected products (Scheme 1).

Several aliphatic and aromatic amines were used for this reaction. Aliphatic amines afforded the products in shorter time compared to aromatic analogues. Aromatic aldehydes carrying either electron-releasing or electron-withdrawing substituents afforded high yields of products. Aliphatic aldehydes could not be used in this procedure because they undergo aldol condensation under the reaction conditions. After optimizing the conditions, the generality toward various amines and benzaldehydes was next explored. The results obtained are listed in Table 1.

Following the obtained results, other derivatives of 2,3dihydroquinazolin-4(1H)-one were synthesized by using different types of amines and aldehydes under aqueous or solvent-free conditions (Scheme 1). Like aliphatic and aromatic amines, heteroaromatic model compounds also afforded the desired products successfully (Table 1).

Silica nanostructures were obtained through a sol-gel method. In this study, the gelation time is defined as the time between pouring the solution in the container and the time at which the solution ceases to discernibly flow under the influence of gravity. The conditions used were shown in Table 2 (experimental section).

The obtained nanostructures were characterized by TEM as shown in Figure 1. This figure shows $40 \mathrm{~nm}$ spheres.

The heteropolyacid $\mathrm{H}_{14}\left[\mathrm{NaP}_{5} \mathrm{~W}_{30} \mathrm{O}_{110}\right]$ in the $\mathrm{SiO}_{2}$ nanoparticle was confirmed by infrared spectroscopy as shown in Figure 2. The asymmetric stretching frequency of the terminal oxygen is observed at $960 \mathrm{~cm}^{-1}$ and the P$\mathrm{O}$ asymmetric stretching frequency is noted at 1080 and $1165 \mathrm{~cm}^{-1}$. The prominent $\mathrm{P}-\mathrm{O}$ bands at 960, 1080, and $1165 \mathrm{~cm}^{-1}$ are consistent with a $\mathrm{C}_{5 \mathrm{~V}}$ symmetry anion. These bands demonstrate that $\mathrm{H}_{14}\left[\mathrm{NaP}_{5} \mathrm{~W}_{30} \mathrm{O}_{110}\right]$ is preserved in the $\mathrm{HPA} / \mathrm{SiO}_{2}$ nanoparticles. In addition, the protonated water of $\mathrm{H}_{14}\left[\mathrm{NaP}_{5} \mathrm{~W}_{30} \mathrm{O}_{110}\right]$ also remained in the nanoparticles at $1730 \mathrm{~cm}^{-1}$. It could be confirmed that the heteropoly acid $\mathrm{H}_{14}\left[\mathrm{NaP}_{5} \mathrm{~W}_{30} \mathrm{O}_{110}\right]$ was successfully immobilized into the $\mathrm{SiO}_{2}$ nanoparticles since the heteropolyacid does not react with $\mathrm{SiO}_{2}$ or with water, but it can remain in the silica nanoparticles without appreciable change of the structures.

Given the importance of such activities, a number of synthetic methods for their synthesis from isatoic anhydride (path 1) and anthranilamide (path 2) have been reported (Schemes 1 and 2). 
Monosubstituted 2,3-dihydroquinazolin-4(1H)-ones were also synthesized successfully using ammonium carbonate as an ammonia source (Scheme 3).

The direct three-component reactions worked well with a variety of arylamines bearing either electron-donating (Table 1, entries 11-17) or -withdrawing groups (Table 1, entries 4, 42-46) and phenethylamine (Table 1, entries 8 and 9). Also, the reactions with arylamines and a range of benzaldehydes carrying either electron-donating or -withdrawing groups on the benzene ring afforded desired products $\mathbf{4} \mathbf{b}-\mathbf{h}$ in high yields. With other primary amines having an aromatic ring, desired products $\mathbf{4 j} \mathbf{j}-\mathbf{l}$ were produced in $78.5-97 \%$ yield (Table 1 , entries $10-12$ ). These reactions provided rapid access to various 2,3-dihydroquinazolin$4(1 H)$-one derivatives (Table $1, \mathbf{4 a}-\mathbf{k})$. We also checked the reusability of the catalyst by separation and reloading in a new run and found that the catalyst could be reused several times without any decrease in the product yield. An example is shown for the reaction of ethylamine with isatoic anhydride and 3-nitrobenzaldehyde, $\mathbf{4 g}$ (Table 1, entry 9). It is well known that some ammonium salts can be applied as the source of ammonia in the synthesis of nitrogen-containing heterocyclic compounds (Scheme 3). Accordingly, 2-aryl substituted 2,3-dihydroquinazolin-4(1H)-ones 7 were synthesized efficiently when ammonium carbonate (6), isatoic anhydride (1), and an aromatic aldehyde $\mathbf{5}$ were treated with Silica-Supported Preyssler Nanoparticles in ethanol under the same reaction conditions (Scheme 3, Table 3).

Some of the synthesized monosubstituted quinazolinones (Table 3, entries 2, 4, 5) have been recognized as potent anticancer compounds. For the preparation of our potential target compounds $\mathbf{2}$ and $\mathbf{3}$, isatoic anhydride was treated with primary amineand terephthaldehyde (4) or glyoxal (5) in the presence of silica-supported preyssler nanoparticles (Scheme 4).

All bis-dihydroquinazolinones synthesized by this pseudo-five-component reaction were reported for the first time and could be considered as potentially biologically active compounds with a quinazolinone core. In addition to the previously mentioned advantages are the simple work-up procedure, which makes this process environmentally friendly, and the easy purification, which requires only filtration of the products followed by recrystallization from ethanol (Table 4).

Different organic solvents were examined for the reaction and we found that water was the solvent of choice (Table 4). Currently the use of non-toxic and environmentally friendly solvents is of much interest. Room temperature ionic liquids are novel solvents with outstanding environmental and technical features, by Wilkes [37]. Tetra- $n$-butylammonium bromide (TBAB) was investigated as solvent for the above reaction. Ethanol proved to be almost as good as water, with ethanol giving a slightly better yield than tetra- $n$ butylammonium bromide. The use of water as a solvent for organic transformations offers several environmental benefits. In many reactions, significant rate enhancements are observed in water compared to organic solvents. This acceleration has been attributed to many factors, including the hydrophobic effect, enhanced hydrogen bonding in the transition state, and the cohesive energy density of water, by Pratt and Pohorille [38]. When the reactions were conducted in water, the expected products were obtained in good yields and with better reaction times compared to organic solvents (Tables 3 and 5).

A plausible mechanism for this reaction is shown in Scheme 5. It is conceivable that the Preyssler heteropolyacid catalysts are coordinated to the oxygen atom of the carbonyl groups in different stages of the reaction activating them for the nucleophilic attack of the amine and amide nitrogen atoms (Scheme 5).

\section{Conclusion}

In conclusion, a simple and environmentally friendly and novel one-pot three-component method for the synthesis of 2,3-dihydroquinazolinones is reported. In this pseudo five-component procedure, six $\mathrm{C}-\mathrm{N}$ bonds are formed in a tandem one-pot process, which is comparable with other important reactions in multicomponent chemistry by Pandey et al. [25]. High yields, ease of work up procedure, use of cheap and commercially available starting materials, convenient manipulation, and mild reaction conditions are the advantages of this new method. We believe that the present methodology addresses the current drive towards green chemistry due to high yields, atomic economy, and reusability of the catalyst. By the reaction of a range of amines and dialdehydes, novel libraries of bisdihydroquinazolinones could be obtained, which would make this method a suitable candidate for combinatorial and parallel synthesis in drug discovery.

\section{References}

[1] P. Salehi, M. Dabiri, M. A. Zolfigol, and M. Baghbanzadeh, "A novel method for the one-pot three-component synthesis of 2,3-dihydroquinazolin-4(1H)-ones," Synlett, no. 7, pp. 1155-1157, 2005.

[2] A. Strecker, "Ueber die künstliche Bildung der Milchsäure und einen neuen, dem Glycocoll homologen Körper," Justus Liebigs Annalen der Chemie, vol. 75, no. 1, pp. 27-45, 1850.

[3] A. Domling, "Recent advances in isocyanide-based multicomponent chemistry," Current Opinion in Chemical Biology, vol. 6, no. 3, pp. 306-313, 2002.

[4] Y. S. Sadanandam, K. R. M. Reddy, and A. B. Rao, "Synthesis of substituted 2,3-dihydro-1-( $\beta$-phenylethyl)-2-aryl and 2,3diaryl-4(1H)-quinazolinones and their pharmacological activities," European Journal of Medicinal Chemistry, vol. 22, no. 2, pp. 169-173, 1987.

[5] G. M. Chinigo, M. Paige, S. Grindrod et al., "Asymmetric synthesis of 2,3-dihydro-2-arylquinazolin-4-ones: methodology and application to a potent fluorescent tubulin inhibitor with anticancer activity," Journal of Medicinal Chemistry, vol. 51, no. 15, pp. 4620-4631, 2008.

[6] T. Graening and H. G. Schmalz, "Total syntheses of colchicine in comparison: a journey through 50 years of synthetic organic chemistry," Angewandte Chemie, vol. 43, no. 25, pp. 3230-3256, 2004.

[7] B. R. Baker, R. E. Schaub, J. P. Joseph, F. J. McEvoy, and J. H. Williams, "An antimalarial alkaloid from hydrangea. XVIII. 
Derivatives of 4-pyrimidone," Journal of Organic Chemistry, vol. 18, no. 2, pp. 133-137, 1953.

[8] J. A. Moore, G. J. Sutherland, R. Sowerby, E. G. Kelly, S. Palermo, and W. Webster, "Reactions of anthranilamide and o-aminoacetophenone with benzil and benzoin," Journal of Organic Chemistry, vol. 34, no. 4, pp. 887-892, 1969.

[9] K. I. Ozaki, Y. Yamada, T. Oine, T. Ishizuka, and Y. Iwasawa, "Studies on 4(1H)-quinazolinones. 5. Synthesis and antiinflammatory activity of $4(1 H)$-quinazolinone derivatives," Journal of Medicinal Chemistry, vol. 28, no. 5, pp. 568-576, 1985.

[10] M. J. Kornet, "Synthesis and anticonvulsant acitivity of 3-alkyl-3,4-dihydro-2(1H)-quinazolinones," Journal of Heterocyclic Chemistry, vol. 29, no. 1, pp. 103-105, 1992.

[11] D. Shi, L. Rong, J. Wang, Q. Zhuang, X. Wang, and H. Hu, "Synthesis of quinazolin-4(3H)-ones and 1,2-dihydroquinazolin$4(3 \mathrm{H})$-ones with the aid of a low-valent titanium reagent," Tetrahedron Letters, vol. 44, no. 15, pp. 3199-3201, 2003.

[12] W. Steiger, T. Kappe, and E. Ziegler, "Synthesen von Heterocyclen, 123. Mitt.: Über Reaktionen des Isatosäureanhydrids mit Anilen," Monatshefte für Chemie, vol. 100, no. 1, pp. 146-149, 1969.

[13] S. W. Li, M. G. Nair, D. M. Edwards et al., "Folate analogues. 35. synthesis and biological evaluation of 1-deaza, 3-deaza, and bridge-elongated analogues of n10-propargyl-5, 8-dideazafolic acid1," Journal of Medicinal Chemistry, vol. 34, no. 9, pp. 27462754, 1991.

[14] C. R. Gorla, N. W. Emanetoglu, S. Liang et al., "Structural, optical, and surface acoustic wave properties of epitaxial $\mathrm{ZnO}$ films grown on (0112) sapphire by metalorganic chemical vapor deposition," Journal of Applied Physics, vol. 85, no. 5, pp. 25952602, 1999.

[15] J. P. Martikainen and H. T. C. Stoof, "Vortex-line solitons in a periodically modulated Bose-Einstein condensate," Physical Review Letters, vol. 93, no. 7, Article ID 070402, 2004.

[16] B. Ding, J. Gong, J. Kim, and S. Shiratori, "Polyoxometalate nanotubes from layer-by-layer coating and thermal removal of electrospun nanofibres," Nanotechnology, vol. 16, no. 6, pp. 785790, 2005.

[17] D. P. Sawant, A. Vinu, N. E. Jacob, F. Lefebvre, and S. B. Halligudi, "Formation of nanosized zirconia-supported 12tungstophosphoric acid in mesoporous silica SBA-15: a stable and versatile solid acid catalyst for benzylation of phenol," Journal of Catalysis, vol. 235, no. 2, pp. 341-352, 2005.

[18] F. F. Bamoharram, M. M. Heravi, M. Roshani, M. Jahangir, and A. Gharib, "Effective direct esterification of butanol by eco-friendly Preyssler catalyst, $\left[\mathrm{NaP}_{5} \mathrm{~W}_{30} \mathrm{O}_{110}\right]^{14-}$," Journal of Molecular Catalysis A, vol. 271, no. 1-2, pp. 126-130, 2007.

[19] F. F. Bamoharram, M. M. Heravi, M. Roshani, A. Gharib, and M. Jahangir, "Catalytic method for synthesis of aspirin by a green, efficient and recyclable solid acid catalyst (Preyssler's anion) at room temperature," Journal of the Chinese Chemical Society, vol. 54, no. 4, pp. 1017-1020, 2007.

[20] A. Müller, F. Peters, M. T. Pope, and D. Gatteschi, "Polyoxometalates: very large clusters-nanoscale magnets," Chemical Reviews, vol. 98, no. 1, pp. 239-271, 1998.

[21] M. H. Alizadeh, H. Razavi, F. Farrash Bamoharram, M. K. Hassanzadeh, R. Khoshnavazi, and F. Mohammadi Zonoz, "Novel catalytic acetylation of alcohols with Preyssler's anion, $\left[\mathrm{NaP}_{5} \mathrm{~W}_{30} \mathrm{O}_{110}\right]^{14-,}$, Kinetics and Catalysis, vol. 44, no. 4, pp. 524-528, 2003.
[22] M. M. Heravi, S. Sadjadi, S. Sadjadi, H. A. Oskooie, R. H. Shoar, and F. F. Bamoharram, "Silica-supported preyssler nanoparticles as new catalysts in the synthesis of $4(3 H)$-quinazolinones," South African Journal of Chemistry, vol. 62, pp. 1-4, 2009.

[23] M. Dabiri, P. Salehi, and M. Baghbanzadeh, "Ionic liquid promoted eco-friendly and efficient synthesis of 2,3-dihydroquinazolin-4(1H)-ones," Monatshefte fur Chemie, vol. 138, no. 11, pp. 1191-1194, 2007.

[24] M. Dabiri, P. Salehi, S. Otokesh, M. Baghbanzadeh, G. Kozehgary, and A. A. Mohammadi, "Efficient synthesis of mono- and disubstituted 2,3-dihydroquinazolin-4(1H)-ones using $\mathrm{KAl}\left(\mathrm{SO}_{4}\right)_{2} \cdot 12 \mathrm{H}_{2} \mathrm{O}$ as a reusable catalyst in water and ethanol," Tetrahedron Letters, vol. 46, no. 36, pp. 6123-6126, 2005.

[25] G. Pandey, R. P. Singh, A. Garg, and V. K. Singh, "Synthesis of Mannich type products via a three-component coupling reaction," Tetrahedron Letters, vol. 46, no. 12, pp. 2137-2140, 2005.

[26] O. A. Maloshitskaya, J. Sinkkonen, V. V. Alekseyev, K. N. Zelenin, and K. Pihlaja, "A comparison of ring-chain tautomerism in heterocycles derived from 2-aminobenzenesulfonamide and anthranilamide," Tetrahedron, vol. 61, no. 30, pp. 7294-7303, 2005.

[27] T. A. Kilroe Smith and H. Stephen, "Syntheses in the quinazolone series-VI. The synthesis of 1:2:3:4-tetrahydro-2-aryl-4oxoquinazolines," Tetrahedron, vol. 1, no. 1-2, pp. 38-44, 1957.

[28] B. Dar, A. K. Sahu, P. Patidar et al., "An easy and efficient protocol for the synthesis of 2,3-dihydroquinazolinones using a low cost and reusable heterogeneous catalyst," American Journal of Chemistry, vol. 2, no. 5, pp. 248-254, 2012.

[29] P. Salehi, M. Dabiri, M. A. Zolfigol, and M. A. B. Fard, "Silica sulfuric acid as an efficient and reusable reagent for crossedaldol condensation of ketones with aromatic aldehydes under solvent-free conditions," Journal of the Brazilian Chemical Society, vol. 15, no. 5, pp. 773-776, 2004.

[30] M. Baghbanzadeh, P. Salehi, M. Dabiri, and G. Kozehgary, "Water-accelerated synthesis of novel bis-2,3-dihydroquinazolin-4(1H)-one derivatives," Synthesis, no. 2, pp. 344-348, 2006.

[31] E. S. Schipper and N. J. Clifton, US 3316269, 1967.

[32] M. A. Zolfigol and M. Safaiee, "Synthesis of 1,4-dihydropyridines under solvent-free conditions," Synlett, no. 5, pp. 827828, 2004.

[33] P. R. Bhalla and B. L. Walworth, EP 0058822, 1982.

[34] M. J. Hour, L. J. Huang, S. C. Kuo et al., "6-alkylamino- and 2,3-dihydro-3' -methoxy-2-phenyl-4-quinazolinones and related compounds: their synthesis, cytotoxicity, and inhibition of tubulin polymerization," Journal of Medicinal Chemistry, vol. 43, no. 23, pp. 4479-4487, 2000.

[35] W. Su and B. Yang, "Reductive cyclization of nitro and azide compounds with aldehydes and ketones promoted by metallic samarium and catalytic amount of iodine," Australian Journal of Chemistry, vol. 55, no. 11, pp. 695-697, 2002.

[36] H. L. Yale and M. Kalkstein, "Substituted 2,3-dihydro-4(IH)quinazolinones. A new class of inhibitors of cell multiplication," Journal of Medicinal Chemistry, vol. 10, no. 3, pp. 334-336, 1967.

[37] J. S. Wilkes, "Properties of ionic liquid solvents for catalysis," Journal of Molecular Catalysis A, vol. 214, no. 1, pp. 11-17, 2004.

[38] L. R. Pratt and A. Pohorille, "Hydrophobic effects and modeling of biophysical aqueous solution interfaces," Chemical Reviews, vol. 102, no. 8, pp. 2671-2692, 2002. 

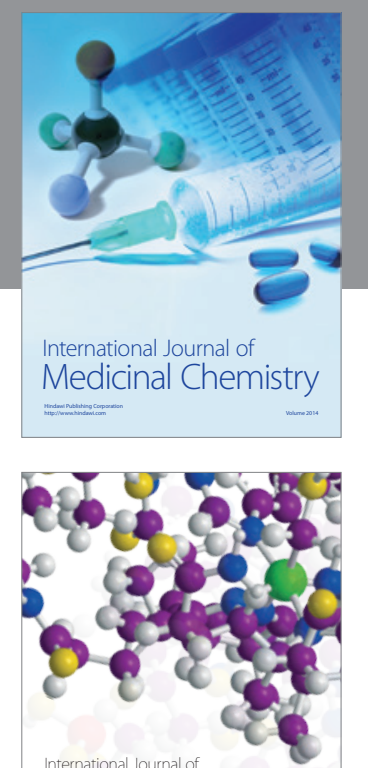

\section{Carbohydrate} Chemistry

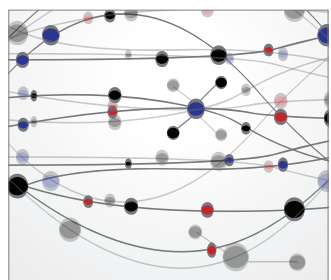

The Scientific World Journal
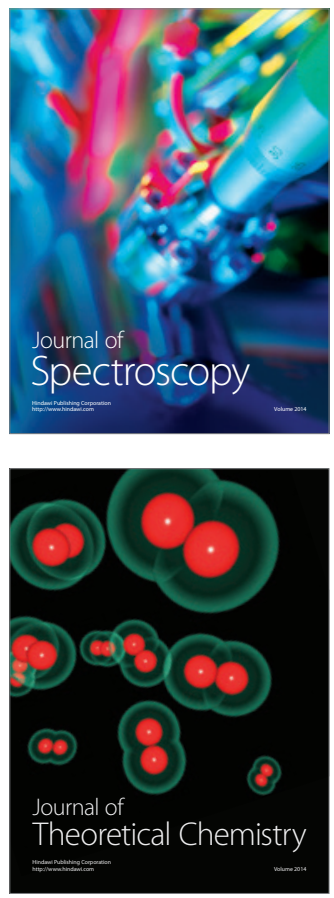
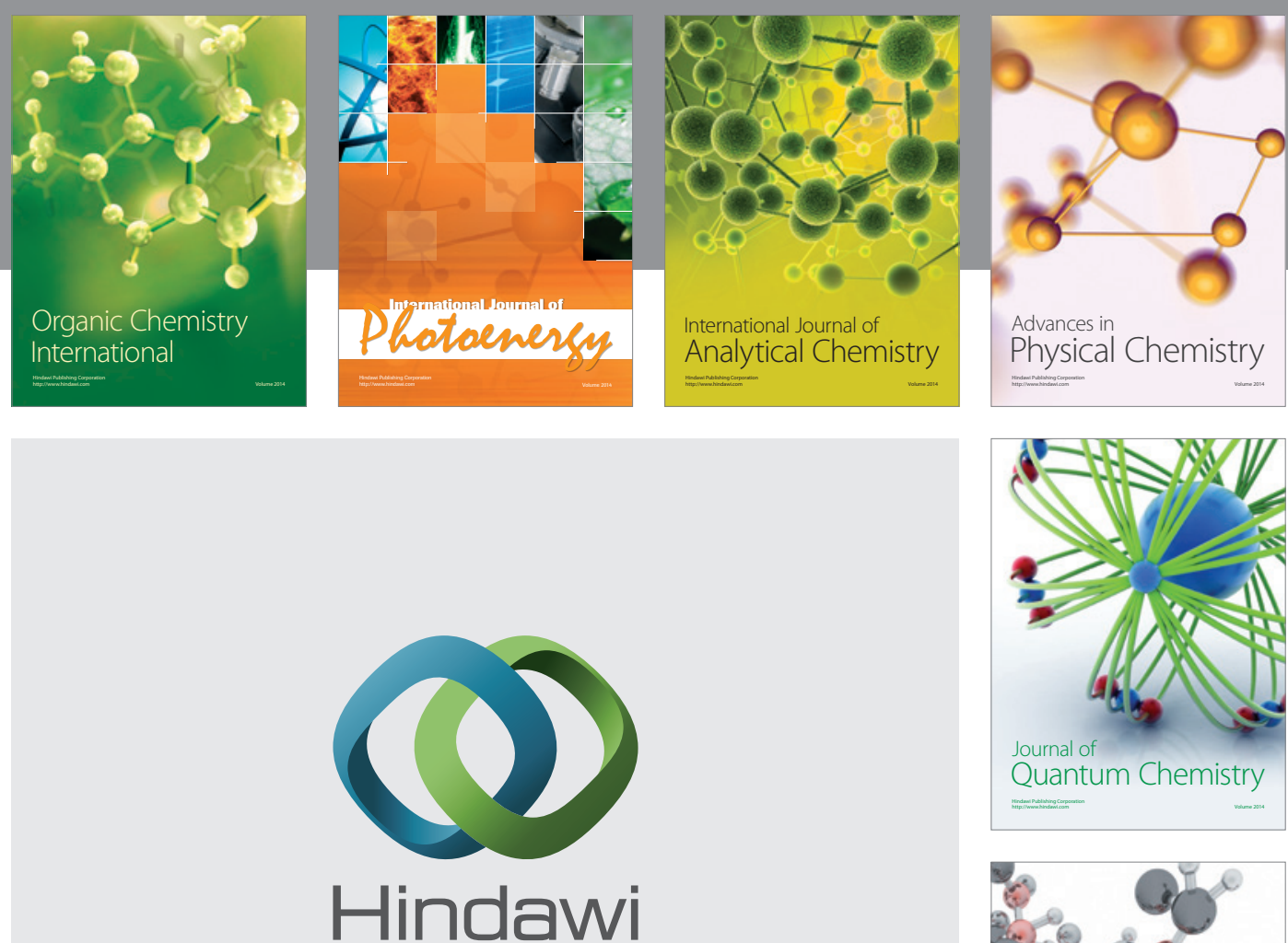

Submit your manuscripts at

http://www.hindawi.com

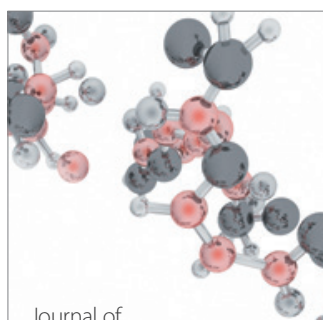

Analytical Methods

in Chemistry

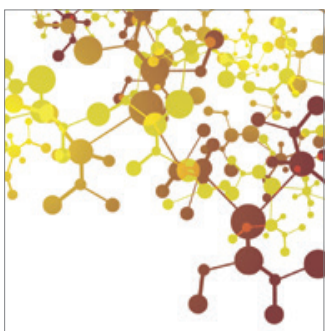

Journal of

Applied Chemistry

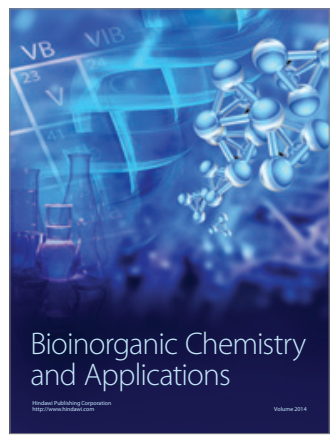

Inorganic Chemistry
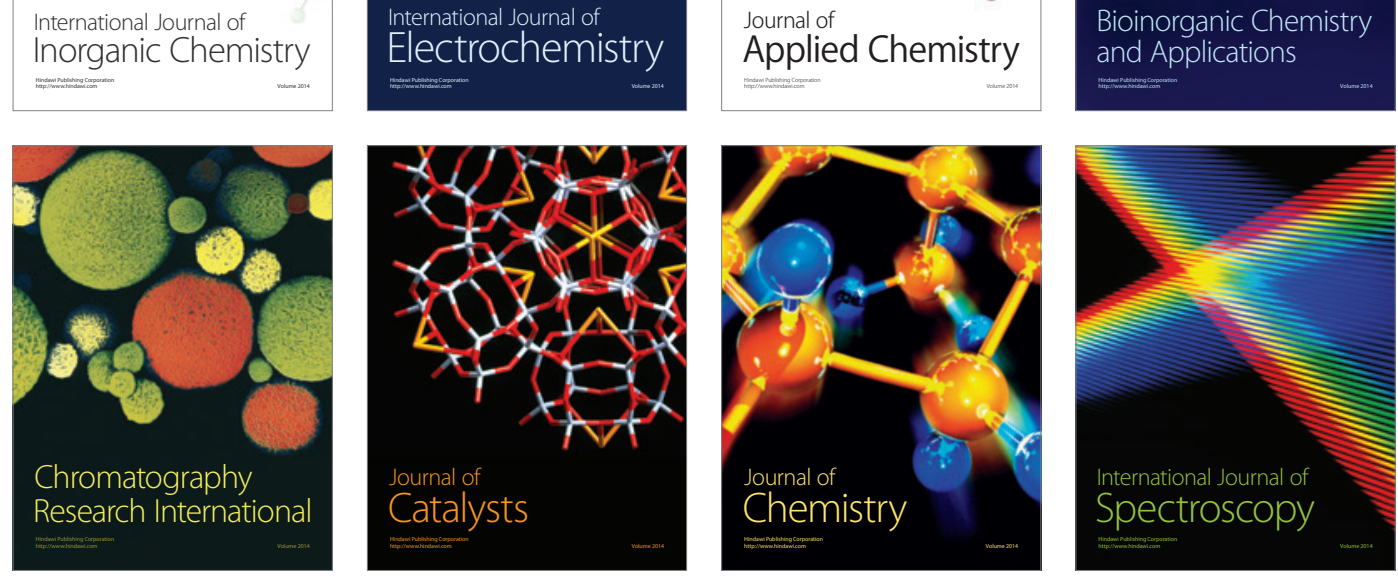